\title{
2-Deoxy-d-glucose inhibits replication of novel coronavirus (SARS-CoV-2) with adverse effects on host cell metabolism
}

\author{
Vikas Malgotra ${ }^{1}$, Varun Sharma ${ }^{2}$ \\ ${ }^{1}$ M.B.B.S, Dermatology Resident, Government Medical College, Jammu, India \\ ${ }^{2}$ Ph.D, Ancient DNA Laboratory, Birbal Sahni Institute of Palaeosciences, Lucknow, India \\ Corresponding author: Vikas Malgotra, e-mail: drvikas2911@yahoo.in
}

\begin{abstract}
The treatment of viral infections is challenging owing to the intricate structure and metabolism of the viruses. In addition, they can highjack host cellular metabolism, mutate and adapt to harsh environmental conditions. The novel coronavirus (SARSCoV-2) displays further resilient attributes, making its eradication even more difficult. SARS-CoV-2 is an enveloped virus whose replication can be targeted by limiting the substrates available for structural incorporation. One such molecule that limits substrate availability and has received much attention lately is 2-Deoxy-d-glucose (2-DG). SARSCoV-2 infection induces glycolysis, impairs mitochondrial function, and damages the infected cells. Administration of 2-DG can inhibit increased glycolytic flux and some other metabolic processes to cause the cessation of viral replication. This article provides a review of the mechanism of action and safety concerns associated with administering 2-DG in the treatment of COVID-19. The drug can have adverse effects on normal cell metabolism since it targets cells non-selectively, possibly in a dosedependent manner. In addition, the drug has limited use in SARS-CoV-2 infection associated with stroke, hypoxic-ischemic encephalopathy, and critical illness.
\end{abstract}

Keywords: 2-Deoxyglucose; Adverse effects; COVID-19; Glutamine; PI3K/Akt 


\section{Introduction}

COVID-19 has emerged as the most rampant and deadliest pandemic of the $21^{\text {st }}$ century. As the numbers of infections continue to ramp up globally, research and drug approvals to find a definite cure for the disease have been expedited. However, there is a lack of drugs effective against the virus.

There is a bidirectional relationship between SARS-CoV-2 infection and increased blood glucose. ${ }^{1,2}$ The cells infected with the virus, like cancer cells, have a high cellular uptake of glucose and its analogs. $.^{3,4} 2-\left[{ }^{18} \mathrm{~F}\right]$ fluoro-2-deoxy-d-glucose $\left({ }^{18} \mathrm{~F}-\mathrm{DG}\right)$ is a radioactive glucose analog widely used as a diagnostic marker for detecting cancer cells by positron emission tomography (PET).

2-Deoxy-d-glucose is another synthetic analog of glucose that acts primarily by inhibiting glycolysis. The novel coronavirus (SARS-CoV-2) upregulates glycolytic and other associated pathways to obtain substrates vital for its structure, function, and replication. It has proven effective in the treatment of cancer, seizures, and viral infections. ${ }^{5,6}$ Since the drug causes complete cessation of replication of SARS-CoV-2 in monocytes infected with the virus, it could help treat COVID-19 along with the commonly used drugs. ${ }^{3}$ However, its therapeutic use is limited due to adverse effects on the cellular metabolism noticed in pre-clinical trials and the limited safety data from clinical trials. ${ }^{7-10}$

\section{Mechanism of action}

2-Deoxy-d-glucose (2-DG) is an analog of glucose (Figure 1) that causes competitive inhibition of the rate-limiting enzyme - Hexokinase. This inhibition induces phosphorylation of glucose to 2-Deoxy-D-glucose-6-phosphate (2-DG-6-P). 2-DG-6-P acts as an allosteric and competitive inhibitor of Hexokinase, and its accumulation also inhibits the enzyme - Phosphoglucoisomerase. ${ }^{11,12}$ Thus, 2-DG can inhibit glycolytic flux, although it can influence multiple other cellular pathways: 


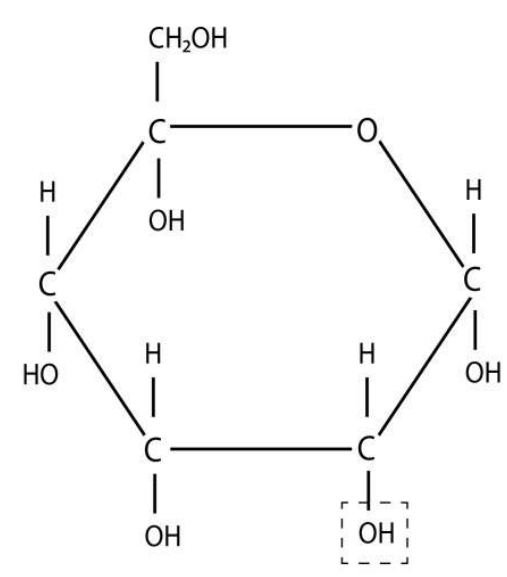

Glucose

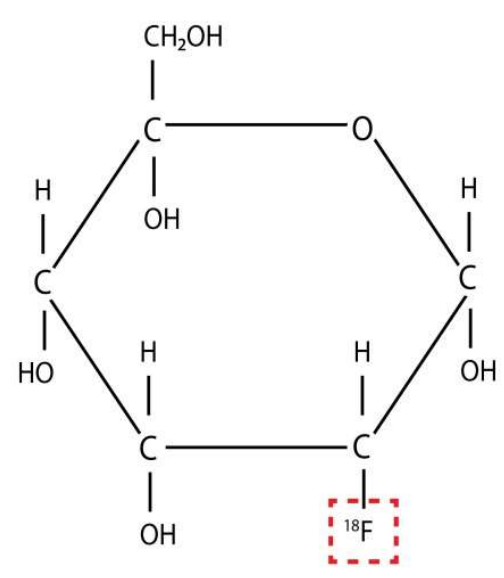

${ }^{18}$ F-Fluorodeoxyglucose

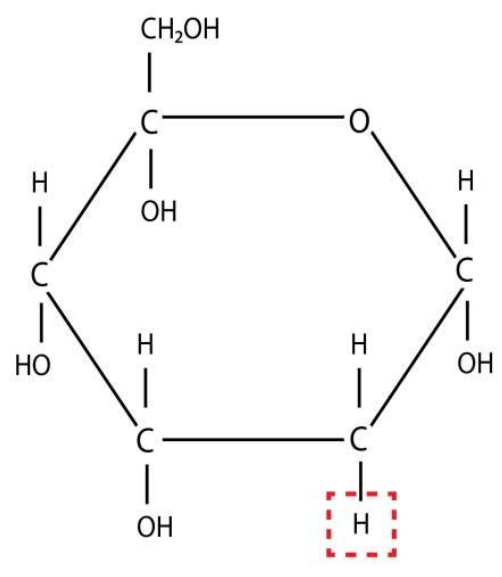

2-Deoxyglucose

Figure 12 2-[18 F]-Fluorodeoxyglucose and 2-Deoxy-d-glucose are structural analogs of glucose that differ on the second carbon in their structure

\section{Inhibition of glycolysis, pentose phosphate pathway, and mitochondrial}

\section{function}

2-DG inhibits the cellular uptake of glucose by glucose transporters (GLUT) and its intracellular utilization by glycolysis. Inhibition of glycolysis depletes ATP, NADH, pyruvate, and nucleotides needed to phosphorylate multiple intermediary metabolites and sustain downstream metabolic pathways.

The depletion of ATP causes catabolic switching while inhibiting the anabolic cellular processes (Figure 2a.). In addition, intracellular ATP depletion is also associated with necrosis as the major pathway of cell death due to membrane instability and extracellular ATP release. These events can result in over-activation of the immune system leading to cytokine storm and acute respiratory distress syndrome (ARDS) in COVID-19. ${ }^{13}$

Glycolysis and apoptosis are closely linked, but the association is poorly understood. Localization of Hexokinase II (HKII) to mitochondria facilitates phosphorylation of glucose by ATP generated inside mitochondria and also has an anti-apoptotic effect by inhibiting the activity of some molecules (Figure 2b.). ${ }^{14}$ Hexokinase inhibition can thus induce apoptosis of the cells through the intrinsic pathway by destabilization of 
mitochondrial complexes. In addition, inhibition of glycolysis results in apoptosis through the extrinsic pathway and autophagy. ${ }^{12}$

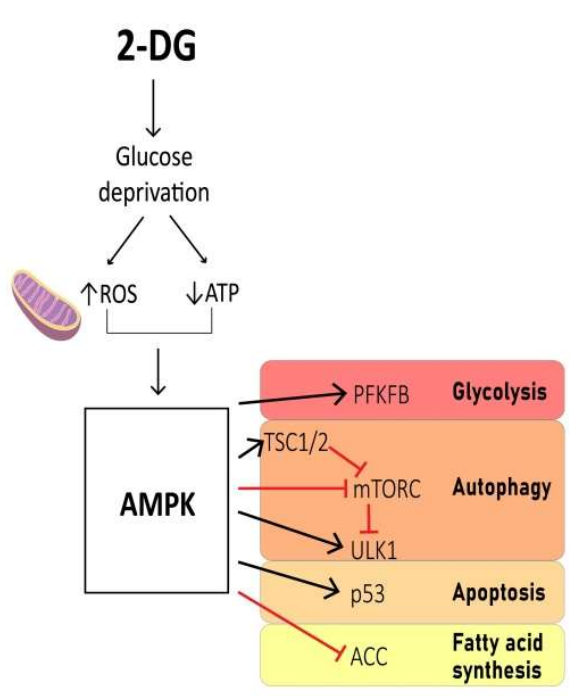

a.

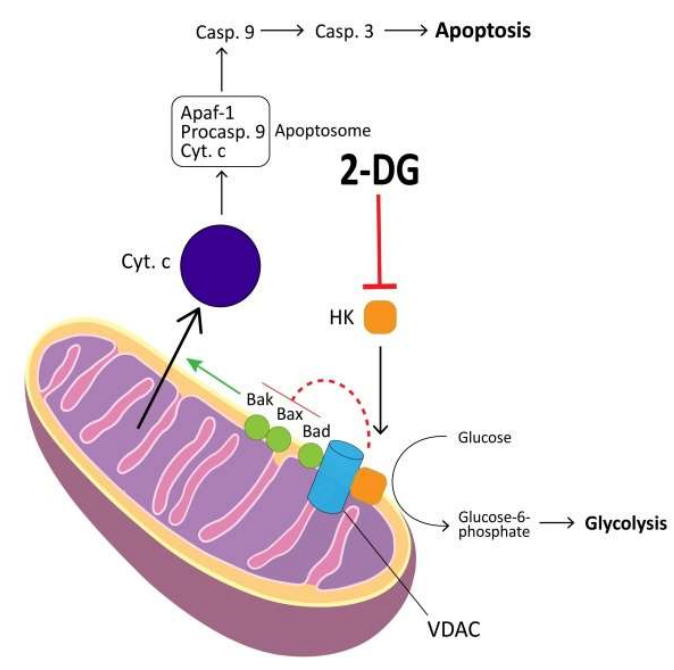

b.

Figure 2 2-DG impacts cell metabolism and induces apoptosis

a. 2-DG causes glucose deprivation to decrease ATP and increase ROS production. As a result, AMPK is activated to increase cell metabolism in favor of increased catabolic activity. It is achieved through increased glycolysis by activating PFKFB and decreased fatty acid synthesis by inhibiting ACC. In addition, activated AMPK increases autophagy through ULK1. It also increases the expression of the tumor suppressor gene p53 to cause apoptosis

b. Hexokinase is mobilized from the cytosol to mitochondria to associate with VDAC. This association inhibits the activity of pro-apoptotic molecules- Bak, Bad, Bax. Activation of these pro-apoptotic molecules can cause apoptosis through the release of cytochrome $c$ into the mitochondria. 2-DG induces apoptosis by inhibiting the anti-apoptotic function of Hexokinase.

ROS: Reactive oxygen species; AMPK: AMP-activated protein kinase; PFKFB: 6-phosphofructo-2-kinase/fructose-2,6bisphosphatase; TSC: Tuberous sclerosis complex; mTORC: mammalian target of rapamycin complex; ULK: Unc-51 like autophagy activating kinase; ACC: Acetyl COA carboxylase; HK: Hexokinase; VDAC: Voltage-dependent anion channel; Bad, Bax, Bak: proapoptotic molecules; Cyt.c: Cytochrome c; Casp.: Caspase

\section{Inhibition of post-translational modification}

2-DG can influence post-translational modification independent of its impact on the inhibition of glycolysis and ATP depletion. In turn, it alters glycosylation-mediated packaging, trafficking, and targeting of vital molecules. Reduced N-linked glycosylation can cause endoplasmic reticulum stress and unfolded protein response mediated autophagy leading to apoptosis. ${ }^{12,13}$ Thus, post-translational modification 
plays a role in apoptotic cell death in an ATP-rich environment even with adequate oxygen.

Thioredoxin interacting protein (TXINP) can induce cell cycle arrest, oxidative stress and downregulate glucose uptake into the cells. ${ }^{15}$ These functions are markedly reduced in tumor cells with increased proliferating activity and glucose utilization. 2DG induces expression of TXINP partly by increasing O-linked glycosylation through a poorly understood mechanism and is independent of the effect of the drug on the Carbohydrate response element (ChoRE).

\section{SARS-CoV-2 infected cells are metabolically analogous to cancer cells}

Cancer cells metabolize glucose to lactate for ATP generation even in the presence of an adequate amount of oxygen (aerobic glycolysis), known as the Warburg effect. This adaptation is attributed to a poorly vascularized, hypoxic tumor environment and impaired mitochondrial electron transport chain. It has a survival benefit for the tumor by supplying ATP to the tumor cells, decreasing apoptotic destruction of the cells, and facilitating tumor spread by lactate-induced degradation of the extracellular matrix. ${ }^{16}$

Monocyte-macrophages at the sites of inflammation are metabolically analogous to the cancer cells. They have impaired mitochondrial function and switch to glycolysis to sustain the increased metabolic demand of their pro-inflammatory phenotype (M1). ${ }^{17}$ In contrast, the anti-inflammatory phenotype (M2) of monocyte-macrophage lineage predominantly depends on oxidative phosphorylation for ATP generation and reparation of the inflammatory damage. However, this phenotype is prevented from activation by mitochondrial dysfunction, AMPK downregulation, and p53 inactivation induced by inflammatory mediators produced in COVID-19. ${ }^{4}$

Aerobic glycolysis is also increased in the virally infected endothelial cells and pneumocytes of the alveolar-capillary barrier. Moreover, alveolar epithelial cells are 
increasingly predisposed to infection with SARS-CoV-2 than endothelial cells or monocytes, especially in the early phase of the infection. ${ }^{18}$

Increased glycolytic activity is a major pathway driving viral replication and the inflammatory response in COVID-19. 3,19 Evidence suggests that monocytes infected with SARS-CoV-2 have increased glycolytic activity associated with the genetic upregulation of enzymes involved in the pathway (Figure 3). ${ }^{3,4}$ This increased glycolytic flux plays a pivotal role in viral entry, replication, and inflammatory response to the infection. It increases gene expression of Angiotensin-converting enzyme 2 (ACE2) mediating intracellular entry of viruses, acts as a carbon source for assimilation of virions, and increases expression of mediators of inflammation and organ damage - IL$1 \beta$, TNF- $\alpha$, IL- 6 , and IFN $\alpha, \beta$, and $\lambda$. These changes are not dependent on mitochondria, as evident from increased expression of ACE2 and inflammatory mediators after oligomycin mediated inhibition of proton channel of ATP synthase and, consequently, electron transport chain and citric acid cycle. ${ }^{3}$

According to a recent study, most of the monocytes derived from peripheral blood of patients with COVID-19 pneumonia were redistributed in favor of increased intermediate/pro-inflammatory cells. They had decreased oxidative phosphorylation and glycolysis. ${ }^{20}$ It is unclear whether these blood cells were harboring SARS-COV-2. These metabolic effects could be exerted distantly by the mediators produced at the sites of inflammation since COVID-19 is associated with low viral detection rates in the blood. ${ }^{21-}$ 23

\section{Association between blood glucose and viral infection}

There is a bidirectional relationship between diabetes and COVID-19. COVID-19 is more common and severe in obese people with insulin resistance and type II diabetes. It is also associated with new-onset diabetes and exacerbation of metabolic complications of pre-existing diabetes - diabetic ketoacidosis and hyperosmolarity. ${ }^{1}$ Hyperglycemia associated with SARS-CoV-2 infection is multifactorial - insulin 
resistance, the stress response to severe illness, pancreatic beta-cell dysfunction, use of steroids for treatment. Amongst these, insulin resistance probably induced by adipose tissue dysfunction is the primary factor underlying hyperglycemia in COVID19. 24

Golgi protein 73 (GP73) is a trans-membrane protein of the Golgi apparatus. Hepatocyte injury is associated with increased expression of the protein and it could serve as a diagnostic and prognostic marker of liver injury in chronic HBV infection. ${ }^{25,26}$ The circulating levels of the protein are approximately $2 x$ elevated in SARS-CoV-2infected patients relative to the reference population $(p<0.0001)$ and its serum concentration correlates with the severity of COVID-19. ${ }^{27}$ It exerts its effect primarily through upregulation of hepatic and renal gluconeogenesis with minimal effect on glycogenolysis. Increased expression of GP73 could be responsible for new onset increase in blood glucose levels associated with SARS-CoV-2 infection. ${ }^{27}$ However, since evidence of raised liver enzymes is present in only 14-53\% cases with COVID$19,{ }^{28}$ GP73 mediated gluconeogenesis can't explain the increased blood glucose levels associated with SARS-COV-2 infection with no hepatocyte injury.

Elevated blood sugar levels cause dysfunction and cell death in diabetes due to increased cellular uptake of glucose. Hexokinase-2 (HK2) mediated glycolytic flux in people with diabetes can impair cellular functions without altering transcription. Increased glycolytic flux can activate hexosamine, protein kinase $\mathrm{C}$, and dicarbonyl stress pathways. ${ }^{29}$

ERK/MAPK and PI3K/AKT/mTOR signaling pathways modulate replication of MERS$\mathrm{CoV}^{30}$. Evidence suggests that SARS-CoV-2 infection is associated with increased blood glucose and activation of these pathways, which provides the substrates for viral replication and affects host cell metabolism (Figure 3) ${ }^{4,31}$ Thus, impairment of the glycolytic pathway can cause a complete cessation of viral replication in cells infected with SARS-CoV-2.4,32 


\section{Effect of 2-DG on viruses}

Since the structure and multiplication of the viruses need substrates and energy derived from the host, exploiting the increased glycolytic flux for these functions could be a significant feature of viral infections. 2-DG can inhibit replicating enveloped viruses like influenza virus, sindbis virus, semliki forest virus, cytomegalovirus, herpes simplex virus, respiratory syncytial virus, and measles virus. ${ }^{33}$

Cytomegalovirus (CMV) causes activation of intracellular calmodulin-dependent kinase kinase 1 (CaMKK1), which mediates increased viral replication and glycolysis. CMVinduced glycolytic induction is not influenced by viral replication, which indicates that it does not need the expression of late proteins dependent on DNA replication. ${ }^{34}$

Inflammatory cells activated in response to viral infection have increased metabolic activity and glucose uptake. Viral infection of the cells can induce and even sustain the increase in blood glucose after the Herpes simplex virus (HSV) no longer persists inside the cells. ${ }^{35}$ It indicates that the viral infection induces genetic alterations in the cell metabolism. CaMKK1 is not needed for viral replication or glycolytic induction caused by the Herpes simplex virus (HSV). Administration of 2-DG impairs glycosylation, inhibiting replication and fusion of the cells infected with the herpes simplex virus (HSV). ${ }^{34,36}$

The glycolytic pathway is central to the generation of citric acid cycle intermediates required to synthesize lipid components for viral entry, replication, and release from the cells. ${ }^{37}$ Thus, 2-DG induced inhibition of glycolysis and post-translational modification has a significant impact in inhibiting the multiplication of the viruses. 


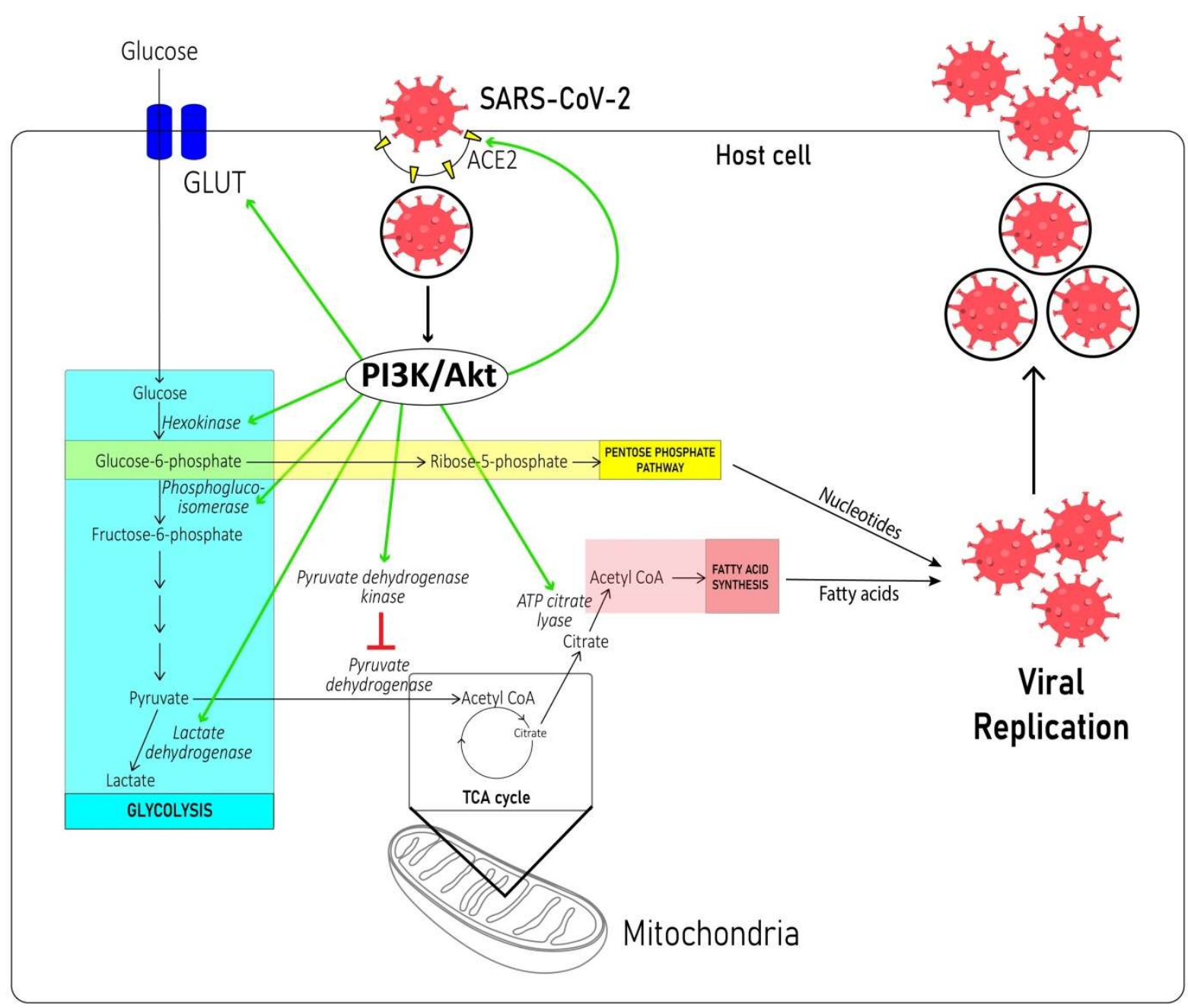

Figure 3 SARS-CoV-2 induced PI3K/Akt pathway has multiple downstream effects.

SARS-CoV2 induces the PI3K/Akt pathway through its effects on the host cell. PI3K/Akt pathway can stimulate glycolysis through increased glucose uptake via GLUT and activation of enzymes- HK, PGI, $\mathrm{LDH}$. Due to activation of LDH and inhibition of PDH, pyruvate is preferentially converted to lactate. Upregulation of the pentose phosphate pathway through increased precursors and fatty acid synthesis through activated ATP citrate lyase provides substrates for viral replication and envelope formation.

\section{2-Deoxy-d-glucose may be less effective in the presence of hypoxia and glutamine}

2-Deoxy-d-glucose (2-DG) preferentially acts on the hypoxic cells with impaired mitochondrial function and increased dependence on glycolysis. In a hypoxic cellular 
environment induced by mitochondrial dysfunction, cell cycle arrest and death occurs at low doses of 2-DG relative to the aerobic cells.(Maher et al., 2004) However, hypoxiamediated cellular effects are not limited to the impairment of glycolysis or mitochondrial function. The hypoxic cells may be rendered resistant to the effects of 2-DG due to increased expression of Hypoxia-inducible factor (HIFa), independent of mitochondrial dysfunction.(Maher et al., 2004; Vaux et al., 2001) Activation of HIFa upregulates the enzymes involved in the glycolytic pathway: hexokinase, aldolase, lactate dehydrogenase. Thus, it can attenuate the inhibitory effect of 2-DG on the glycolytic pathway and the resultant cell death due to ATP/ substrate depletion or allosteric feedback (Figure 2).(Maher et al., 2004; Pajak et al., 2020)

Another concern with limiting glycolytic flux for inhibition of viral assembly and replication is the availability of alternate substrates to execute these functions. Glutamine is the most abundant naturally occurring amino acid in the body and provides substrates for the citric acid cycle, with 2-DG exerting no influence on cellular glutamine transport or metabolism. Glutamine plays a role in the pathogenesis and impairment of immune response associated with COVID-19.(Bharadwaj et al., 2021). However, recent evidence suggests oxidative glutamine metabolism in the mitochondria is reduced in SARS-CoV-2 infection. ${ }^{38}$

\section{Administration of 2-Deoxy-D-Glucose can affect normal functioning cells}

2-DG has established good safety in many pre-clinical studies except a few. ${ }^{7,12,39}$ Limited data from human studies show that it is well tolerated. ${ }^{8-10}$ However, since the drug can interfere with the normal cellular metabolism, its use in humans is concerning due to possible associated adverse effects that can arise after extrapolating the data obtained from the pre-clinical studies. The drug can adversely affect the neurological, cardiovascular, endocrinological, and hematological systems. 


\section{a. Neurological effects}

2-DG can confer neuroprotection to central nervous system neurons but causes increased cell death in the presence of hypoxia. Microglial cells serve as the macrophages of the central nervous system (CNS). 2-DG inhibits glycolysis in the microglia in vitro, and the resulting ATP depletion causes cell death. This selective effect of 2-DG on microglia is due to their increased dependence on glycolysis, mainly upon activation. On the contrary, neurons are predominantly dependent on mitochondria to meet their ATP requirements. ${ }^{40,41}$ Thus, neurons are relatively unaffected by the effects of the drug in animal models of the diseases with microglial activation associated neuronal loss - Alzheimer's disease, Parkinson's disease, stroke, trauma, meningitis, epilepsy. However, the neuroprotection is not absolute, and increased neuronal death is observed in pathological states associated with hypoxia stroke, vascular dementia, and trauma. ${ }^{41}$

The prevalence of neurological involvement in severe COVID-19 varies from $3.5 \%$ to $84 \%$ in different studies. ${ }^{42-44}$ In a prospective observational study on 4,491 patients with COVID-19, 606 (13.5\%) developed neurological symptoms. ${ }^{45}$ The most common neurological diagnoses were - metabolic encephalopathy $(6.8 \%)$, seizure $(1.6 \%)$, stroke $(1.9 \%)$, and hypoxic injury (1.4\%). There were no cases of meningitis or myelitis, and RT-PCR tests conducted on CSF were negative. This indicates that CNS involvement with COVID-19 infection is an indirect consequence of systemic impairment caused by SARS-CoV-2. COVID-19 associated seizures can be theoretically treated by the administration of 2-DG. ${ }^{5}$ However, the drug can have adverse effects in case of stroke and hypoxia-mediated brain injury.

The drug can have adverse effects on the peripheral nervous system. A clinical trial administering the glycolysis inhibitor dichloroacetate was prematurely terminated due to increased mortality and adverse effects of the drug on the peripheral nervous system. ${ }^{46}$ This adverse effect could be attenuated by providing an alternate source of ATP via ketogenic or Atkin's diet. ${ }^{5,47}$ However, since 2-DG administration results in a similar metabolic profile as caloric restriction, it is associated with poor clinical outcomes if used 
for treating critically ill patients for whom caloric restriction is absolutely contraindicated. 47

\section{b. Cardiovascular effects}

Chronic ingestion of 20 to $300 \mathrm{mg} / \mathrm{kg}$ 2-DG increased the risk of cardiotoxicity, pheochromocytoma, and mortality in rats. ${ }^{7}$ The cardiotoxicity was reversible after 60 days, and a decline in cardiac function could be predicted by monitoring the trend of biomarkers like NT-proBNP and BNP levels. ${ }^{39}$ Furthermore, it was associated with abnormal ECG changes like T-wave flattening and QT prolongation, which were nonserious and reversible within 24 hours. $^{8}$

\section{c. Endocrinological effects}

Hypoglycemia-associated autonomic failure: Administration of 2-DG is associated with cellular glucose deprivation (glucoprivation) and can impair resulting central and autonomic compensatory mechanisms (Figure 4). The secretion of glucocorticoids induced by stress-response possibly mediates this hypoglycemia-associated autonomic failure, which may even be fatal. ${ }^{48} 2-D G$ induced compensatory hyperglycemia can be prevented by the administration of beta-blockers.

Administration of 2-DG was associated with increased prevalence of pheochromocytoma in a pre-clinical study conducted in rats. ${ }^{7}$ The tumorigenesis is thought to be mediated by the alteration of HIFa pathway and mitochondrial impairment induced by the administration of 2-DG.

\section{d. Hematological effects}

Platelet activation is associated with increased glycolysis and glycogenolysis. The 2-DG associated decrease in glucose uptake and metabolism can impair platelet aggregation and degranulation in vitro. ${ }^{49}$

\section{Adverse effects associated with other drugs inhibiting glycolysis}


Arsenic compounds, 2-Deoxy-D-glucose (2-DG), 3-Bromopyruvate (3-BrPA), oxamate, and Dichloroacetate (DCA) inhibit glycolysis and ATP generation.

Arsenic compounds inhibit glycolysis indirectly by impairing mitochondrial respiration. ${ }^{50}$ They cause acute and chronic toxicity, and arsenic poisoning is fatal within 1-4 days of exposure, depending on the dose. Acute abdominal pain, pulmonary edema, respiratory failure, cardiomyopathy, coagulation abnormalities, chronic peripheral neuropathy are the significant concerns with the toxicity. ${ }^{51}$

3-BrPA impairs glycolysis by covalent modification and inhibition of Hexokinase (HKII). This modification triggers its dissociation from mitochondria leading to the release of apoptosis-inducing factor (AIF) from the mitochondria to cytosol and eventual cell death. In addition, the drug can inhibit multiple other cellular pathways. ${ }^{52}$

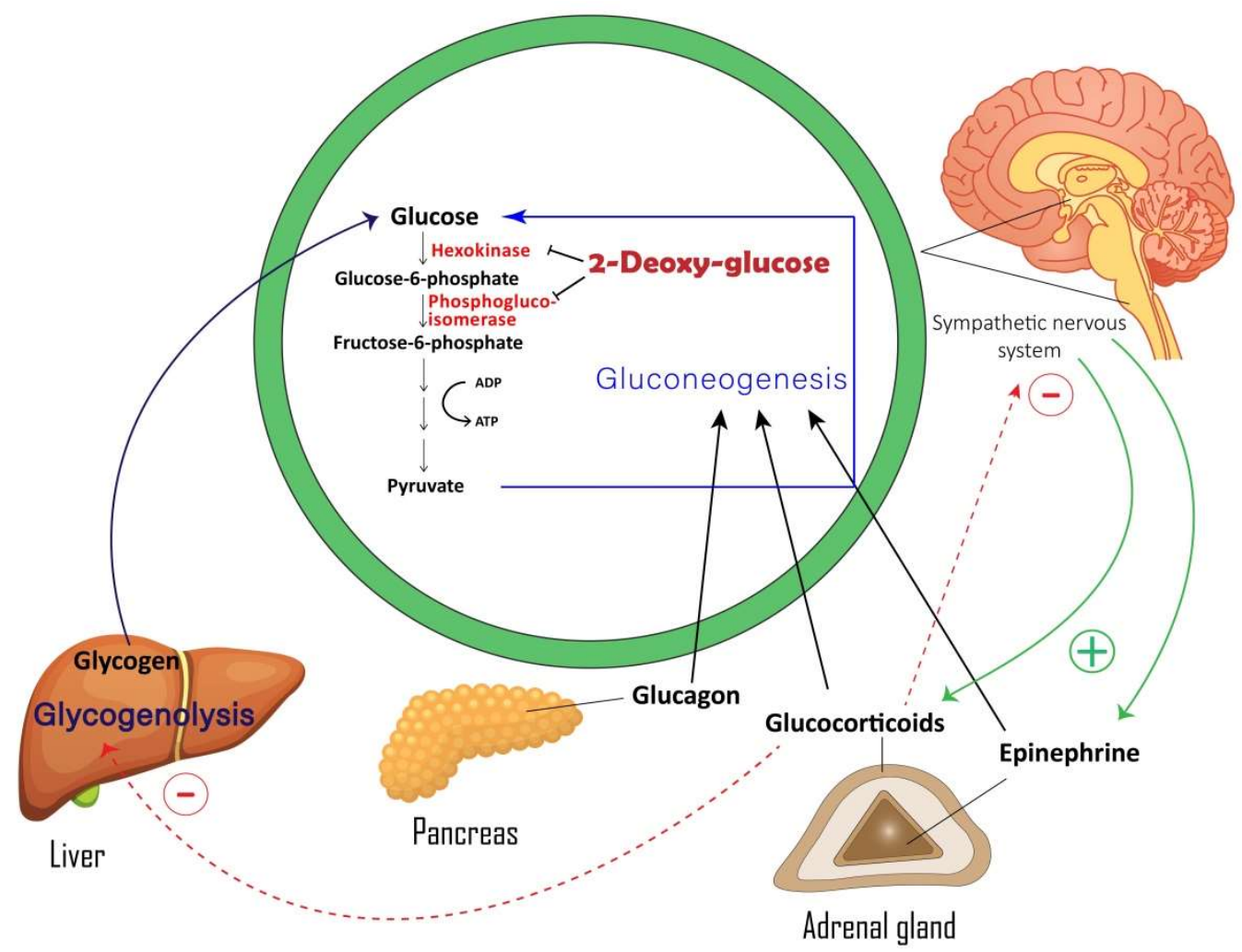

Figure 4 2-DG can cause autonomic impairment in patients with Type 2 diabetes

Intracellular glucopenia in the hypothalamic and brain stem neurons elicits appetite and secretion of glucagon, glucocorticoids, and catecholamines resulting in hyperglycemia. Circulating glucocorticoids also inhibit the sympathetic nervous system and hypothalamic-pituitary-adrenal (HPA) axis by acting on hindbrain catecholaminergic neurons as well as by inhibiting gluconeogenesis. These changes, in turn, result in inhibition of the glucopenia-induced compensatory increase in appetite and hyperglycemia. ${ }^{48}$ 
Administration of Dichloroacetate (DCA) for the treatment of Mitochondrial Encephalopathy Lactic Acidosis and Stroke (MELAS) was studied in a clinical trial conducted on 30 patients by Kaufmann et al. ${ }^{46}$ Almost all (19 out of 22 ) the patients receiving DCA either initially or after crossover from the placebo arm developed peripheral neuropathy, which was at least partially reversible. The drug was concluded to have a low therapeutic index and ineffective in the treatment of MELAS. However, patients in the study were proportionately older and diabetic than the studies conducted earlier; these two factors may have contributed to the development of peripheral neuropathy associated with the drug administration.

\section{Conclusion}

2-Deoxyglucose (2-DG) exerts effects through mechanisms dependent and independent of glycolysis inhibition. It has achieved good results in inhibiting viral replication in studies conducted in vitro. However, there are theoretical risks related to decreased efficacy of the drug in the presence of hypoxia and the availability of alternate substrates for viral replication and assembly. In addition, the drug administration can be associated with adverse effects on the heart, neurons, adrenal medulla, and platelets, especially since it has not been widely studied for therapeutic use in many clinical trials. The drug can also have limited clinical use in treating stroke, hypoxic-ischemic encephalopathy, and critical illness since it can deplete the energy stores available for cell metabolism. Therefore, 2-DG should be cautiously used in low doses and short duration after due consideration of the clinical status and risk-benefit ratio on a case by case basis. In view of the availability of alternate drugs with widely studied mechanisms of action and known side-effect profile for the treatment of the disease, experimental drugs like 2-DG should only be included in the standard treatment protocols after conducting multiple double-blind clinical trials achieving predefined primary endpoints. 
Author Contributions: Conceived and designed the article: VM; Wrote the manuscript: VM and VS; Revised the manuscript: VM and VS. All authors have read and agreed to the published version of the manuscript.

Funding: This research received no external funding.

Acknowledgments: Not applicable.

Conflicts of Interest: The authors declare no conflict of interest.

Copyright disclaimer: No figures have been reproduced or adapted. All research works were properly attributed wherever necessary.

\section{References}

1. Rubino F, Amiel SA, Zimmet $\mathrm{P}$, et al. New-Onset Diabetes in Covid-19. N Engl J Med. 2020;383(8):789-790. doi:10.1056/nejmc2018688

2. Lim S, Bae JH, Kwon HS, Nauck MA. COVID-19 and diabetes mellitus: from pathophysiology to clinical management. Nat Rev Endocrinol. 2021;17:11-30. doi:10.1038/s41574-020-00435-4

3. Codo AC, Davanzo GG, Monteiro L de B, et al. Elevated Glucose Levels Favor SARS-CoV-2 Infection and Monocyte Response through a HIF-1a/GlycolysisDependent Axis. Cell Metab. 2020;32(3):437-446.e5. doi:10.1016/j.cmet.2020.07.007

4. Icard P, Lincet $\mathrm{H}, \mathrm{Wu} Z$, et al. The key role of Warburg effect in SARS-CoV-2 replication and associated inflammatory response. Biochimie. 2021;180:169-177. doi:10.1016/j.biochi.2020.11.010

5. Kossoff EH, Zupec-Kania BA, Auvin S, et al. Optimal clinical management of children receiving dietary therapies for epilepsy: Updated recommendations of the International Ketogenic Diet Study Group. Epilepsia Open. 2018;3(2):175-192. doi:10.1002/epi4.12225

6. $\mathrm{Xi} \mathrm{H}$, Kurtoglu M, Lampidis TJ. The wonders of 2-deoxy-d-glucose. IUBMB Life. 2014;66(2):110-121. doi:10.1002/iub.1251

7. Minor RK, Smith DL, Sossong AM, et al. Chronic ingestion of 2-deoxy-d-glucose 
induces cardiac vacuolization and increases mortality in rats. Toxicol Appl Pharmacol. 2010;243(3):332-339. doi:10.1016/j.taap.2009.11.025

8. Burckhardt $D$, Stalder GA. Cardiac changes during 2 Deoxy $D$ glucose test: a study in patients with selective vagotomy and pyloroplasty. Digestion. 1975;12(1):1-8. doi:10.1159/000197647

9. Singh D, Banerji AK, Dwarakanath BS, et al. Optimizing cancer radiotherapy with 2-deoxy-D-glucose: Dose escalation studies in patients with glioblastoma multiforme. Strahlentherapie und Onkol. 2005;181(8):507-514. doi:10.1007/s00066-005-1320-z

10. Thomas DG, Duthie HL. Use of 2 deoxy-D-glucose to test for the completeness of surgical vagotomy. Gut. 1968;9(1):125-128. doi:10.1136/gut.9.1.125

11. Vishwanatha A, D'Souza CJM. Multifaceted effects of antimetabolite and anticancer drug, 2-deoxyglucose on eukaryotic cancer models budding and fission yeast. IUBMB Life. 2017;69(3):137-147. doi:10.1002/iub.1599

12. Pajak B, Siwiak E, Sołtyka M, et al. 2-Deoxy-D-Glucose and its analogs: From diagnostic to therapeutic agents. Int J Mol Sci. 2020;21(1):234. doi:10.3390/ijms21010234

13. Xi H, Kurtoglu M, Liu H, et al. 2-Deoxy-d-glucose activates autophagy via endoplasmic reticulum stress rather than ATP depletion. Cancer Chemother Pharmacol. 2011;67(4):899-910. doi:10.1007/s00280-010-1391-0

14. Kim J, Dang C V. Multifaceted roles of glycolytic enzymes. Trends Biochem Sci. 2005;30(3):142-150. doi:10.1016/j.tibs.2005.01.005

15. Hong SY, Hagen T. 2-Deoxyglucose induces the expression of thioredoxin interacting protein (TXNIP) by increasing O-GIcNAcylation - Implications for targeting the Warburg effect in cancer cells. Biochem Biophys Res Commun. 2015;465(4):838-844. doi:10.1016/j.bbrc.2015.08.097

16. Sanchez WY, McGee SL, Connor T, et al. Dichloroacetate inhibits aerobic glycolysis in multiple myeloma cells and increases sensitivity to bortezomib. $\mathrm{Br} \mathrm{J}$ Cancer. 2013;108(8):1624-1633. doi:10.1038/bjc.2013.120

17. Kramer PA, Ravi S, Chacko B, Johnson MS, Darley-Usmar VM. A review of the mitochondrial and glycolytic metabolism in human platelets and leukocytes: 
Implications for their use as bioenergetic biomarkers. Redox Biol. 2014;2(1):206210. doi:10.1016/j.redox.2013.12.026

18. Chilosi M, Poletti V, Ravaglia C, et al. The pathogenic role of epithelial and endothelial cells in early-phase COVID-19 pneumonia: victims and partners in crime. Mod Pathol. Published online April 21, 2021:1-12. doi:10.1038/s41379021-00808-8

19. Ajaz S, McPhail MJ, Singh KK, et al. Mitochondrial metabolic manipulation by SARS-CoV-2 in peripheral blood mononuclear cells of patients with COVID-19. Am J Physiol - Cell Physiol. 2021;320(1):C57-C65.

doi:10.1152/AJPCELL.00426.2020

20. Gibellini L, De Biasi S, Paolini A, et al. Altered bioenergetics and mitochondrial dysfunction of monocytes in patients with COVID-19 pneumonia. EMBO Mol Med. 2020;12:e13001. doi:10.15252/emmm.202013001

21. Huang $Y$, Chen $S$, Yang Z, et al. SARS-CoV-2 viral load in clinical samples from critically ill patients. Am J Respir Crit Care Med. 2020;201(11):1435-1438. doi:10.1164/rccm.202003-0572LE

22. Ling Y, Xu SB, Lin YX, et al. Persistence and clearance of viral RNA in 2019 novel coronavirus disease rehabilitation patients. Chin Med J (Engl). 2020;133(9):1039-1043. doi:10.1097/CM9.0000000000000774

23. Wölfel R, Corman VM, Guggemos W, et al. Virological assessment of hospitalized patients with COVID-2019. Nature. 2020;581(7809):465-469.

doi:10.1038/s41586-020-2196-x

24. Reiterer M, Rajan M, Gómez-Banoy N, et al. Hyperglycemia in Acute COVID-19 is Characterized by Adipose Tissue Dysfunction and Insulin Resistance. medRxiv. Published online March 26, 2021:2021.03.21.21254072.

doi:10.1101/2021.03.21.21254072

25. Xu Z, Liu L, Pan X, et al. Serum golgi protein 73 (GP73) is a diagnostic and prognostic marker of chronic HBV liver disease. Med (United States). 2015;94(12):e659. doi:10.1097/MD.0000000000000659

26. Kladney RD, Bulla GA, Guo L, et al. GP73, a novel Golgi-localized protein upregulated by viral infection. Gene. 2000;249(1):53-65. doi:10.1016/S0378- 
1119(00)00136-0

27. Wan L, Yang H, Li H, et al. GP73 is a glucogenic hormone regulating SARS-CoV2-induced hyperglycemia. medRxiv. Published online May 3, 2021:2021.04.30.21256060. doi:10.1101/2021.04.30.21256060

28. Zhang C, Shi L, Wang FS. Liver injury in COVID-19: management and challenges. Lancet Gastroenterol Hepatol. 2020;5(5):428-430. doi:10.1016/S2468-1253(20)30057-1

29. Rabbani N, Thornalley PJ. Hexokinase-2 Glycolytic Overload in Diabetes and Ischemia-Reperfusion Injury. Trends Endocrinol Metab. 2019;30(7):419-431. doi:10.1016/j.tem.2019.04.011

30. Kindrachuk J, Ork B, Hart BJ, et al. Antiviral potential of ERK/MAPK and $\mathrm{PI} 3 \mathrm{~K} / \mathrm{AKT} / \mathrm{mTOR}$ signaling modulation for Middle East respiratory syndrome coronavirus infection as identified by temporal kinome analysis. Antimicrob Agents Chemother. 2015;59(2):1088-1099. doi:10.1128/AAC.03659-14

31. Karam BS, Morris RS, Bramante CT, et al. mTOR inhibition in COVID-19: A commentary and review of efficacy in RNA viruses. J Med Virol. 2021;93(4):18431846. doi:10.1002/jmv.26728

32. Bojkova D, Klann K, Koch B, et al. Proteomics of SARS-CoV-2-infected host cells reveals therapy targets. Nature. 2020;583:469. doi:10.1038/s41586-020-2332-7

33. Kang HT, Hwang ES. 2-Deoxyglucose: An anticancer and antiviral therapeutic, but not any more a low glucose mimetic. Life Sci. 2006;78(12):1392-1399. doi:10.1016/j.lfs.2005.07.001

34. McArdle J, Schafer XL, Munger J. Inhibition of Calmodulin-Dependent Kinase Kinase Blocks Human Cytomegalovirus-Induced Glycolytic Activation and Severely Attenuates Production of Viral Progeny. J Virol. 2011;85(2):705-714. doi:10.1128/jvi.01557-10

35. Varanasi SK, Donohoe D, Jaggi U, Rouse BT. Manipulating Glucose Metabolism during Different Stages of Viral Pathogenesis Can Have either Detrimental or Beneficial Effects. J Immunol. 2017;199(5):1748-1761. doi:10.4049/jimmunol.1700472

36. Thaker SK, Ch'ng J, Christofk HR. Viral hijacking of cellular metabolism. BMC 
Biol. 2019;17(1):59. doi:10.1186/s12915-019-0678-9

37. Mansouri K, Rastegari-Pouyani M, Ghanbri-Movahed M, Safarzadeh M, Kiani S, Ghanbari-Movahed Z. Can a metabolism-targeted therapeutic intervention successfully subjugate SARS-COV-2? A scientific rational. Biomed Pharmacother. 2020;131:110694. doi:10.1016/j.biopha.2020.110694

38. Mullen PJ, Garcia G, Purkayastha A, et al. SARS-CoV-2 infection rewires host cell metabolism and is potentially susceptible to mTORC1 inhibition. Nat Commun. 2021;12(1):1876. doi:10.1038/s41467-021-22166-4

39. Terse PS, Joshi PS, Bordelon NR, et al. 2-Deoxy- d -Glucose (2-DG)-Induced Cardiac Toxicity in Rat. Int J Toxicol. 2016;35(3):284-293. doi:10.1177/1091581815624397

40. Abe N, Nishihara T, Yorozuya T, Tanaka J. Microglia and Macrophages in the Pathological Central and Peripheral Nervous Systems. Cells. 2020;9(9):2132. doi:10.3390/cells9092132

41. Vilalta A, Brown GC. Deoxyglucose prevents neurodegeneration in culture by eliminating microglia. J Neuroinflammation. 2014;11:58. doi:10.1186/1742-209411-58

42. Helms J, Kremer S, Merdji H, et al. Neurologic Features in Severe SARS-CoV-2 Infection. N Engl J Med. 2020;382(23):2268-2270. doi:10.1056/nejmc2008597

43. Mao L, Jin $\mathrm{H}$, Wang M, et al. Neurologic Manifestations of Hospitalized Patients with Coronavirus Disease 2019 in Wuhan, China. JAMA Neurol. 2020;77(6):683690. doi:10.1001/jamaneurol.2020.1127

44. Romero-Sánchez CM, Díaz-Maroto I, Fernández-Díaz E, et al. Neurologic manifestations in hospitalized patients with COVID-19: The ALBACOVID registry. Neurology. 2020;95(8):e1060-e1070. doi:10.1212/WNL.0000000000009937

45. Frontera JA, Sabadia S, Lalchan R, et al. A Prospective Study of Neurologic Disorders in Hospitalized Patients With COVID-19 in New York City. Neurology. 2021;96(4):e575-e586. doi:10.1212/WNL.0000000000010979

46. Kaufmann P, Engelstad K, Wei Y, et al. Dichloroacetate causes toxic neuropathy in MELAS: A randomized, controlled clinical trial. Neurology. 2006;66(3):324-330. doi:10.1212/01.wnl.0000196641.05913.27 
47. Voss M, Lorenz NI, Luger AL, Steinbach JP, Rieger J, Ronellenfitsch MW. Rescue of 2-deoxyglucose side effects by ketogenic diet. Int J Mol Sci. 2018;19(8):2462. doi:10.3390/ijms19082462

48. Sanders NM, Ritter S. Acute 2DG-induced glucoprivation or dexamethasone abolishes 2DG-induced glucoregulatory responses to subsequent glucoprivation. Diabetes. 2001;50(12):2831-2836. doi:10.2337/diabetes.50.12.2831

49. George MJ, Bynum J, Nair P, et al. Platelet biomechanics, platelet bioenergetics, and applications to clinical practice and translational research. Platelets. 2018;29(5):431-439. doi:10.1080/09537104.2018.1453062

50. Ralph SJ. Arsenic-based antineoplastic drugs and their mechanisms of action. Met Based Drugs. 2008;2008. doi:10.1155/2008/260146

51. Ratnaike RN. Acute and chronic arsenic toxicity. Postgrad Med J. 2003;79(933):391-396. doi:10.1136/pmj.79.933.391

52. Sun Y, Liu Z, Zou X, et al. Mechanisms underlying 3-bromopyruvate-induced cell death in colon cancer. J Bioenerg Biomembr. 2015;47(4):319-329. doi:10.1007/s10863-015-9612-1 